\title{
DATA-DRIVEN HUMAN ACTIVITY RECOGNITION IN SMART ENVIRONMENTS
}

\author{
Marina Marjanović-Jakovljević, \\ Angelina Njeguš \\ Singidunum University, \\ 32 Danijelova Street, Belgrade, Serbia
}

\begin{abstract}
:
Many applications of human activity recognition like healthcare, security etc. show how human activity recognition is important in everyday life. In this paper, we compare different machine learning algorithms like Naïve Bayes (NB), One R (1R) rule, Zero R (0R) rule, J 48 trees, Random Forest (RF) and Random Tree (RT) applied on sensor-based human activity recognition in a home environment. We show that Random Forest achieves better performance in terms of correctly classified instances comparing to other algorithms, while application of $0 \mathrm{R}$ rules algorithm achieves significantly the worst performance. Additionally, in order to reduce the dimensionality of the algorithm, we applied wrapper method using the same classifier in the attribute selection. It is shown that using the wrapper method the performance of the classification in terms of correctly classified instances is not significantly changed, while it shows much better performance in terms of algorithm complexity. After calculating accuracy of each algorithm, we calculate accuracy for each activity classified by each classifier.
\end{abstract}

Key words:

activity recognition, machine learning, sensors, classification, wrapper.

\section{INTRODUCTION AND THEORETICAL BACKGROUND}

The Internet evolved into Internet of Things (IoT), from processors embedded into the computers to processors and sensors embedded almost in every "thing" i.e. in any device. IoT, by market segment, can be classified into three broader categories, such as health self-tracking and personal environment monitoring, smart homes/buildings, and transportation/ automotive applications (Swan, 2012). Activity recognition is the foundation of these areas as it enables a wide range of computing applications (e.g. elder care and health applications). In some papers it is shown that it is possible to detect a large range of activities (Bao et al, 2004) (Huynh et al, 2007; Lester et al, 2006). Since human activities are complex and highly diverse, the goal of activity recognition is to recognize common activities in daily life (Kim et al, 2010). However, recognizing complex human activities is still challenging area, especially when dealing with concurrent or interleaved activities.

Activity recognition can be defined as the process that includes (a) adequate sensors to monitor and capture a user's behavior according to environment state change, and (b) system for collecting, storing, processing, and analyzing perceived information, in order to create activity models for
Correspondence:

Angelina Njeguš

e-mail:

anjegus@singidunum.ac.rs 
developing algorithms that will infer activities from sensor data (Chen et al, 2012). Activity recognition can be visionbased, sensor-based, data-driven or knowledge-driven.

Vision-based activity recognition uses visual sensing devices (e.g. video cameras) that generate video sequences or video digitized data. On the other hand, sensor based activity recognition uses wearable sensors or smart phones (attached to an actor), or dense sensing (attached to objects) that generate time series of state changes or various parameter values that are processed through some probabilistic or statistical analysis methods (Chen et al, 2012). With the expansion of mobile computing, wearable sensors receive more attention. Sensor-based are more convenient for smart environments, such as smart homes, smart hospitals, smart buildings, etc. In Anguita et al. (2012), a system for activity recognition is presented using Smartphone inertial sensors. Since mobile phones are limited in terms of energy and computing power, a novel hardware-friendly approach for multiclass classification is proposed. This method is based on Support Vector Machine and exploits fixed-point arithmetic for computational cost reduction.

Data-driven activity recognition creates user activity models from existing large datasets of user behaviors using data mining and machine learning techniques, and then uses the learnt activity models to infer activities (Gu et al, 2011; Okeyo et al, 2012). However, it is difficult to apply learnt activity models generally to all people. In Ordonez et al. (2012), the use of two machine learning algorithms, Artificial Neural Network and Support Vector Machines, within the framework of Hidden Markov Model, in order to perform activity recognition in a home environment, is described. A knowledge-driven activity recognition construct activity models based on rich prior knowledge. Hybrid approaches combine knowledge-driven and machine learning to formulate activity models (Okeyo et al, 2012).

In this paper different classification models applied on sensor-based dataset in home environment are compared. The aim is to identify the algorithm that achieves better performance for sensor-based activity recognition. Since all algorithms analyzed in numerous papers are extremely complex, this paper proposes wrapper methods to reduce the dimensionality of each method.

This paper is organized in three parts. The first part describes seven machine learning methods and their comparative advantages for the activity recognition problem. The second part describes datasets with selected activities and type of sensors. The third part presents the results of experiment and compares the performance between selected methods.

\section{CLASSIFICATION MODELS DESCRIPTION}

To represent and recognize the activities based on the optimal features, six different machine learning algorithms like NB, 1R, 0R, J 48 trees, RF and RT were applied to sensor-based data in terms of CCI and number of attributes were selected by a wrapper method.

\section{Naive Bayes Model}

Since all of the attributes contribute equally and independently to the decision, we can apply Naive Bayes (NB) method explained by John et al. (1995). Probability of event $H$ with given evidence $E$ is presented as

$$
P_{r}(H \mid E)=\frac{P_{r}(E \mid H) P_{r}(H)}{P_{r}(E)}
$$

Where $P_{r}(H)$ presents Prior probability and $P_{r}(H \mid E)$ presents Posterior probability of event $H$. In our model, $H$ presents a user activity that we want to identify, and evidence $E$ presents an instance in our dataset. Evidence splits into independent parts

$$
P_{r}(E \mid H)=P_{r}\left(E_{1} \mid H\right) P_{r}\left(E_{2} \mid H\right) \ldots P_{r}\left(E_{n} \mid H\right)
$$

Where particular evidences, or attributes $E_{1}, E_{2} \ldots E_{n}$, are statistically independent.

\section{One R rule Model}

This method is explained by Holt (1993). This method, the same as NB Model, relies on Frequency Table (Kohavi, 1995). It is based on using the minimum-error attribute for prediction, discretizing numeric attributes.

\section{Zero $R$ rule Model}

The same as NB Model, OR model is based on Frequency Table. This is the simplest classification method which relies on the target and ignores the predictors. It predicts the mean (for a numeric class) or the mode (for a nominal class) constructing a frequency table and selecting its most frequent value.

\section{J 48 Trees Model}

J48 model is one of the Decision Trees models, a hierarchical data structure based on conquer strategy. This classification model is explained by Quinlan (1993). The idea is to select which attribute to divide on at the root node, and then create a branch for each possible attribute value. Then, in order to make the selection, the procedure is recursively repeated for each branch, selecting an attribute at each node, using only instances 
that reach that node. The objective is to get the smallest tree, and top-down tree induction methods use different approaches. The most used approach to produce pure nodes is an information theory-based approach founded by Claude Shannon (1948).

\section{Random Forest}

Since the combination of classification models increases the classification accuracy, Random Forest (RF) model is proposed (Breiman, 2001). It works as a large collection of forest of the correlated random decision trees.

\section{Random Tree Model}

This model is based on constructing a tree that considers $\mathrm{K}$ randomly chosen attributes at each node. A Random Tree (RT) model is explained by Aldous in 1991. RT model associated with random graphs is also explained by Aldous in 1990 .

\section{Wrapper-based Approach}

In order to decrease data dimensionality, it will be interesting to select the most effective features from our feature vector. In Kohavi et al. (1997), Wrapper method for feature selection is described. The flowchart of wrapper-based approach in this paper is presented in Figure 1.

This method is based on the evaluation of the attribute sets by using a learning scheme. Cross validation is used in order to estimate the accuracy of the learning scheme for a set of attributes. In order to compare performance with and without subset extraction and find a subset, the evaluator will use the same classifier as in training set.

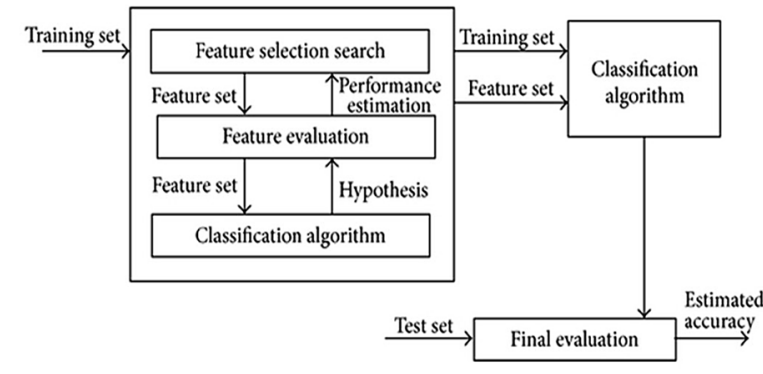

Figure 1. Flowchart of wrapper-based approach to feature subset selection (Kohavi et at, 1997)

\section{DATA SETS DESCRIPTION}

In order to validate this testing, we use 407 instances from Ordonez A dataset generated by Kasteren (2013). This dataset comprises activities of daily living (ADL) performed by a user in home environment. Datasets were generated by a set of simple state-change sensors. The wireless sensor network (WSN) was measuring passive infrared sensors to detect motion, reed switches for opening or closing of doors or cupboards, and float sensors for measuring the toilet being flushed.

Instances were described by description, sensor event (feature) and ADL (label). Features were recorded using a WAN and data were labelled manually (UCI, 2013). Nine different ADLs, included as labels, were considered: "Leaving", "Toileting", "Showering", "Grooming", "Sleeping", "Breakfast", "Lunch", "Snack", and "Spare time".

Table I shows the number of separate instances per activity in the dataset. Table II, Table III and Table IV show attributes that are considered for this test as location, place and sensor type, respectively.

\begin{tabular}{clc}
\hline $\mathrm{N}_{0}$ & Label & Count \\
\hline 1 & Sleeping & 15 \\
\hline 2 & Toileting & 19 \\
\hline 3 & Grooming & 111 \\
\hline 4 & Showering & 14 \\
\hline 5 & Breakfast & 70 \\
\hline 6 & Spare_Time/TV & 80 \\
\hline 7 & Leaving & 31 \\
\hline 8 & Lunch & 49 \\
\hline 9 & Snack & 18 \\
\hline & Table I. Activities count \\
\end{tabular}

\begin{tabular}{clc}
\hline $\mathrm{N}_{0}$ & Label & Count \\
\hline $\mathbf{1}$ & Bed & 15 \\
\hline $\mathbf{2}$ & Cabinet & 15 \\
\hline $\mathbf{3}$ & Basin & 70 \\
\hline $\mathbf{4}$ & Toilet & 45 \\
\hline $\mathbf{5}$ & Shower & 14 \\
\hline $\mathbf{6}$ & Fridge & 56 \\
\hline $\mathbf{7}$ & Cupboard & 34 \\
\hline $\mathbf{8}$ & Toaster & 14 \\
\hline $\mathbf{9}$ & Cooktop & 13 \\
\hline $\mathbf{1 0}$ & Microwave & 20 \\
\hline $\mathbf{1 1}$ & Seat & 80 \\
\hline $\mathbf{1 2}$ & Maindoor & 31 \\
\hline
\end{tabular}

Table II. Location of sensors count 


\begin{tabular}{clc}
\hline $\mathrm{N}_{0}$ & Label & Count \\
\hline $\mathbf{1}$ & Bedroom & 15 \\
\hline $\mathbf{2}$ & Bathroom & 144 \\
\hline $\mathbf{3}$ & Kitchen & 137 \\
\hline $\mathbf{4}$ & Living & 80 \\
\hline $\mathbf{5}$ & Entrance & 31 \\
\hline
\end{tabular}

Table III. Place count

\begin{tabular}{clc}
\hline $\mathrm{N}_{0}$ & Label & Count \\
\hline $\mathbf{1}$ & Pressure & 95 \\
\hline $\mathbf{2}$ & Magnetic & 136 \\
\hline $\mathbf{3}$ & PIR & 97 \\
\hline $\mathbf{4}$ & Flush & 45 \\
\hline $\mathbf{5}$ & Electric & 34 \\
\hline
\end{tabular}

Table IV. Type of sensors count

\section{EXPERIMENTS AND RESULTS}

This experiment was performed using WEKA (Waikato Environment for Knowledge Analysis) tool, developed at the University of Waikato in New Zealand. This software contains large spectrum of tools such as: data pre-processing, classification, regression, clustering, association rules, and visualization. The purpose of this paper is to compare six different machine learning algorithms like NB, 1R, 0R, J 48 trees, RF and RT applied on sensor-based human activity recognition in a home environment in terms of correctly classified instances (CCI) and number of attributes (NA) selected by a wrapper method. After calculating accuracy of each algorithm, it is important to calculate accuracy for each activity classified by each classifier.

In the first part of experiment, we compare classifiers in terms of CCI applied on the entire data set, that has 4 attributes. In the second part of the experiment, we used wrapper method in order to reduce the dimensionality of data. The results are provided with a 10 -fold crossvalidation.

\begin{tabular}{lcccccc}
\hline & \multicolumn{6}{c}{ Classifier } \\
\cline { 2 - 7 } & NB & $1 \mathrm{R}$ & $0 \mathrm{R}$ & $\mathrm{J} 48$ & $\mathrm{RF}$ & $\mathrm{RT}$ \\
\hline CCI in original data set (\%) & 78,1 & 77,8 & 27,3 & 76,6 & 79,6 & 77,9 \\
\hline CCI with wrapper (\%) & 77,1 & 77,9 & 27,3 & 76,9 & 76,6 & 77,9 \\
\hline NA with wrapper & 3 & 1 & 0 & 3 & 1 & 1 \\
\hline
\end{tabular}

Table V. Clasiffiers comparison

\begin{tabular}{lccc}
\hline & \multicolumn{3}{c}{ Metric } \\
\cline { 2 - 4 } & $\mathrm{P}$ & $\mathrm{R}$ & $\mathrm{ROC}$ \\
\hline Sleeping & 1 & 1 & 1 \\
\hline Toileting & 0 & 0 & 0,854 \\
\hline Grooming & 0,854 & 1 & 0,968 \\
\hline Showering & 1 & 1 & 1 \\
\hline Breakfast & 0,681 & 0,457 & 0,926 \\
\hline Spare_Time/TV & 1 & 1 & 1 \\
\hline Leaving & 1 & 1 & 1 \\
\hline Lunch & 0,389 & 0,714 & 0,885 \\
\hline Snack & 0 & 0 & 0,915 \\
\hline
\end{tabular}

Table VI. Deatailed accuracy by class- NB

\begin{tabular}{lccc}
\hline & \multicolumn{3}{c}{ Metric } \\
\cline { 2 - 4 } & $\mathrm{P}$ & $\mathrm{R}$ & $\mathrm{ROC}$ \\
\hline Sleeping & 1 & 1 & 1 \\
\hline Toileting & 0 & 0 & 0,5 \\
\hline Grooming & 0,854 & 1 & 0,968 \\
\hline Showering & 1 & 1 & 1 \\
\hline Breakfast & 0,597 & 0,614 & 0,764 \\
\hline Spare_Time/TV & 1 & 1 & 1 \\
\hline Leaving & 1 & 1 & 1 \\
\hline Lunch & 0,354 & 0,469 & 0,676 \\
\hline Snack & 0 & 0 & 0,5 \\
\hline
\end{tabular}

Table VII. Deatailed accuracy by class $-1 \mathrm{R}$

\begin{tabular}{lccc}
\hline & \multicolumn{3}{c}{ Metric } \\
\cline { 2 - 4 } & $\mathrm{P}$ & $\mathrm{R}$ & $\mathrm{ROC}$ \\
\hline Sleeping & 0 & 0 & 0,415 \\
\hline Toileting & 0 & 0 & 0,474 \\
\hline Grooming & 0,273 & 1 & 0,492 \\
\hline Showering & 0 & 0 & 0,41 \\
\hline Breakfast & 0 & 0 & 0,497 \\
\hline Spare_Time/TV & 0 & 0 & 0,497 \\
\hline Leaving & 0 & 0 & 0,482 \\
\hline Lunch & 0 & 0 & 0,489 \\
\hline Snack & 0 & 0 & 0,451 \\
\hline
\end{tabular}

Table VIII. Deatailed accuracy by class- 0 R 


\begin{tabular}{lccc}
\hline & \multicolumn{3}{c}{ Metric } \\
\cline { 2 - 4 } & $\mathrm{P}$ & $\mathrm{R}$ & $\mathrm{ROC}$ \\
\hline Sleeping & 1 & 1 & 1 \\
\hline Toileting & 0 & 0 & 0,849 \\
\hline Grooming & 0,854 & 1 & 0,966 \\
\hline Showering & 1 & 1 & 1 \\
\hline Breakfast & 0,51 & 0,714 & 0,9 \\
\hline Spare_Time/TV & 1 & 1 & 1 \\
\hline Leaving & 1 & 1 & 1 \\
\hline Lunch & 0,282 & 0,224 & 0,874 \\
\hline Snack & 0 & 0 & 0,894 \\
\hline
\end{tabular}

Table IX. Deatailed accuracy by class -J48

\begin{tabular}{lccc}
\hline & \multicolumn{3}{c}{ Metric } \\
\cline { 2 - 4 } & $\mathrm{P}$ & $\mathrm{R}$ & $\mathrm{ROC}$ \\
\hline Sleeping & 1 & 1 & 1 \\
\hline Toileting & 0 & 0 & 0,85 \\
\hline Grooming & 0,854 & 1 & 0,966 \\
\hline Showering & 1 & 1 & 1 \\
\hline Breakfast & 0,662 & 0,614 & 0,927 \\
\hline Spare_Time/TV & 1 & 1 & 1 \\
\hline Leaving & 1 & 1 & 1 \\
\hline Lunch & 0,417 & 0,612 & 0,886 \\
\hline Snack & 0 & 0 & 0,916 \\
\hline Tabl Xette
\end{tabular}

Table X. Deatailed accuracy by class - RF

\begin{tabular}{lccc}
\hline & \multicolumn{3}{c}{ Metric } \\
\cline { 2 - 4 } & $\mathrm{P}$ & $\mathrm{R}$ & $\mathrm{ROC}$ \\
\hline Sleeping & 1 & 1 & 1 \\
\hline Toileting & 0 & 0 & 0,849 \\
\hline Grooming & 0,854 & 1 & 0,966 \\
\hline Showering & 1 & 1 & 1 \\
\hline Breakfast & 0,597 & 0,614 & 0,925 \\
\hline Spare_Time/TV & 1 & 1 & 1 \\
\hline Leaving & 1 & 1 & 1 \\
\hline Lunch & 0,354 & 0,404 & 0,883 \\
\hline Snack & 0 & 0 & 0,915 \\
\hline
\end{tabular}

Table XI. Deatailed accuracy by class -RT
According to Table V, we can conclude that in the case when we use the entire data set, RF algorithm achieves better performance in terms of CCI comparing to other algorithms, while application of Zero $\mathrm{R}$ rules algorithm achieves significantly the worst performance. On the other hand, when wrapper approach is applied, RT has better performance in terms of CCI comparing to RT, while $1 \mathrm{R}$ achieves higher $\mathrm{CCI}$ comparing to NB. Observing data dimensionality, NB and J48 show higher complexity than other algorithms.

In Table V-Table XI, detailed accuracy for each activity, for each classifier used in this paper is presented. Precision $(\mathrm{P})$ is calculated as proportion of instances that are correctly classified divided by the total instances classified as that class. While Recall (R) is calculated as a ratio of the proportion of instances classified as a given class and the actual total in that class.

Receiver operating characteristics (ROC) graphs are very useful for classifiers comparison in machine learning and data mining research (Fawcett, 2005).

According to table VI, activities such as: "Leaving", "Sleeping", and "Spare time" have the best performance, while "Breakfast", and "Lunch" show the worst performance when NB classifier is applied.

In table VII is demonstrated that activities such as: "Sleeping", "Leaving", "Showering", and "Spare time" have best performance in terms of $\mathrm{P}, \mathrm{R}$ and ROC when 1 $\mathrm{R}$ classifier is applied, while activities such as "Toileting" and "Snack" result in significantly worst performance.

Table VIII shows that when 0 R classifier is applied, the best performance shows activity "Grooming". However, it still has very low precision of 0,273.

According to table IX, activities such as: "Leaving", "Sleeping", "Spare time" and "Showering" have the best performance, while activities such as "Breakfast", "Lunch" and "Snack", whose recognition requires magnetic sensors activation, show the worst performance when J48 classifier is applied.

In tables $\mathrm{X}$ and $\mathrm{XI}$ accuracy by class when RF and RT classifier is applied is shown respectively. They show similar result where "Sleeping", "Showering", "Spare time" and "Leaving" result in best performance, while "Snack" and "Lunch" activities have very low P and R.

\section{CONCLUSION AND FUTURE WORK}

According to the results obtained, we can see that order in performance of applied classifiers in terms of CCI is different when wrapper method is applied. In 
case when we use the entire data set for the purpose of human activity recognition, RF classifier shows the best performance, while in case when wrapper approach is applied, RT shows the best performance compared to other classifiers used in this paper.

Additionally, it is shown that is possible to improve the system performance in the human activity recognition problems, using the wrapper method for reducing the dimensionality of the data. In further research, it would be interesting to compare those classifiers on all five data sets described by Kasteren (2013) as: "KasterenA", "KasterenB", "KasterenC", “OrdonezA" and "OrdonezB".

\section{REFERENCES}

Aldous, D. (1990) A Random Tree Model Associated with Random Graphs. Random Structures and Algorithms, 1(4), 382-402.

Aldous, D. (1991) The Continuum Random Tree. I. The Annals of Probability, 19(1), 1-28.

Anguita, D., Ghio, A., Oneto, L., Parra, X., Reyes-Ortiz, J.L. (2012) Human Activity Recognition on Smartphones using a Multiclass Hardware-Friendly Support Vector Machine. Ambient Assisted Living and Home Care. Vol. 7657 of the series Lecture Notes in Computer Science. Springer Berlin Heidelberg, pp: 216-223.

Bao, L. and Intille, S.S. (2004) Activity Recognition from User Annotated Acceleration Data. Pervasive Computing. Vol. 3001 of the series Lecture Notes in Computer Science. Springer Berlin Heidelberg, pp: 1-17.

Breiman, L. (2001) Random Forests. Machine Learning, 45(1), 5-32.

Chen, L., Hoey, J., Nugent, C., Cook, D. Yu, Z. (2012) Sensor-based Activity Recognition. IEEE Transactions on Systems, Man, and Cybernetics, Part C: Applications and Reviews. May, 2012, pp. 790-808.

Fawcett, T. (2006) An introduction to ROC analysis. Pattern Recognition Letters - Special issue: ROC Analysis in pattern recognition, 27(8), 861-874.

Gu, T., Wang, L., Wu, Z., Tao, X., Lu, J. (2011) A Pattern Mining Approach to Sensor-Based Human Activity Recognition. IEEE Transactions on Knowledge and Data Engineering, 23(9), 1359-1372.

Holte, R.C. (1993) Very simple classification rules perform well on most commonly used datasets. Machine Learning, 11(1), 63-90.

Huynh, T., Blanke, U. and Schiele, B. (2007) Scalable Recognition of Daily Activities from Wearable Sensors. In Proc. of International Workshop on Locationand Context-Awareness (LoCA), LNCS, Vol. 4718. Springer. pp: 50-67.
John, G. H., Langley, P. (1995) Estimating Continuous Distributions in Bayesian Classifiers. In Proceedings of the Eleventh Conference on Uncertainty in Artificial Intelligence - UAI'95. Morgan Kaufmann Publishers Inc. San Francisco, CA, USA, pp: 338-345.

Kasteren, T. (2010) Datasets for Activity Recognition. Available at: https://sites.google.com/site/tim0306/ datasets (accessed: 3.02.2016).

Kim, E. Helal, S., Cook, D. (2010) Human Activity Recognition and Pattern Discovery. IEEE Pervasive Computing, 9(1), 48-53.

Kohavi, R. (1995) The Power of Decision Tables. In Proceedings of the European Conference on Machine Learning, pp. 174-189.

Kohavi, R., John, G.H. (1997) Wrappers for feature subset selection. Artificial Intelligence, 97(1-2), pp: 273-324.

Lester, J., Choudhury, T., Borriello, G. (2006) A Practical Approach to Recognizing Physical Activities. In Proc. of the 4th International Conference on Pervasive Computing. Springer-Verlag Berlin, Heidelberg, pp: 1-16.

Okeyo, G., Chen, L., Wang, H., Sterritt, R. (2012) A Knowledge-Driven Approach to Composite Activity Recognition in Smart Environments. Ubiquitous Computing and Ambient Intelligence. Vol. 7656 of the series Lecture Notes in Computer Science. Springer Berlin Heidelberg. pp. 322-329.

Ordóñez, F.J., Toledo, P., Sanchis, A. (2013) Activity Recognition Using Hybrid Generative/Discriminative Models on Home Environments Using Binary Sensors. Sensors. Vol. 13, Iss. 5, pp: 5460-5477.

Quinlan, J. R. (1993) C4.5: Programs for Machine Learning. Morgan Kaufmann Publishers, San Francisco, CA, USA.

Shannon, C. E. (1948) A Mathematical Theory of Communication. Bell System Technical Journal. Vol. 27, Iss. 3, pp:379-423.

Swan, M. (2012) Sensor Mania! The Internet of Things, Wearable Computing, Objective Metrics, and the Quantified Self 2.0 Journal of Sensor and Actuator Networks. Vol. 1, pp 217-253.

UCI (2013) Activities of Daily Living (ADLs) Recognition Using Binary Sensors Data Set. Machine Learning Repository - Center for Machine Learning and Intelligent Systems. Available at: https://archive.ics.uci. edu/ml/datasets/Activities+of+Daily+Living+(ADL s)+Recognition+Using+Binary+Sensors (accessed: 5.01.2016). 